\title{
A cavity-enhanced broadband photonic Rabi oscillation
}

\author{
Rikizo Ikuta, ${ }^{1,2}$ Toshiki Kobayashi, ${ }^{1,2}$ Tomohiro Yamazaki, ${ }^{1}$ Nobuyuki Imoto, ${ }^{2}$ and Takashi Yamamoto ${ }^{1,2}$ \\ ${ }^{1}$ Graduate School of Engineering Science, Osaka University, Toyonaka, Osaka 560-8531, Japan \\ ${ }^{2}$ Center for Quantum Information and Quantum Biology, Osaka University, Osaka 560-8531, Japan
}

\begin{abstract}
A coherent coupling among different energy photons provided by nonlinear optical interaction is regarded as a photonic version of the Rabi oscillation. Cavity enhancement of the nonlinearity reduces energy requirement significantly and pushes the scalability of the frequency-encoded photonic circuit based on the photonic Rabi oscillation. However, confinement of the photons in the cavity severely limits the number of interactable frequency modes. Here we demonstrate a wide-bandwidth and efficient photonic Rabi oscillation achieving full-cycle oscillation based on a cavity-enhanced nonlinear optical interaction with a monolithic integration. We also show its versatile manipulation beyond the frequency degree of freedom such as an all-optical control for polarizing photons with geometric phase. Our results will open up full control accessible to synthetic dimensional photonic systems over wide frequency modes as well as a large-scale photonic quantum information processing.
\end{abstract}

Rabi oscillation, which is a cyclic rotation between coherently-coupled two atomic levels driven by an optical field, is one of the most fundamental building block in atomic physics, and has been used for numerious technologies such as atomic clocks [1], sensors 2], quantum communication [3] and computing [4, 5]. In a photonic system, the coherent two-level system is implemented by two distinct photonic frequencies coupled by nonlinear optical interaction with a pump light. Recently, such a photonic Rabi oscillation has been applied to a single photon known as quantum frequency conversion (QFC) [6] in quantum information processing, which can create a coherent superposition of the frequency modes in the single photon. Apart from atomic systems using naturally-determined energy levels, the photonic systems use virtual levels determined by the pump frequency corresponding to the difference of the two frequencies (Fig. 1 (a)). This feature allows for interaction of densely-embedded optical frequency modes over a wide range, and offers flexible manipulation of photonic frequencies that form the inherently-equipped high dimensional Hilbert space.

So far, several experimental demonstrations of quantum operations on the frequency-encoded qubits based on optical nonlinearities have been performed [7-9] aiming at frequency-domain photonic quantum information processing such as universal quantum computation based on multi-stage nonlinear optical interaction with the photonic Rabi oscillation [10 12]. Typically, the full Rabi cycle in $\chi^{(2)}$-based QFC corresponding to the so-called $2 \pi$ pulse needs over a watt-class continuous wave (cw) pump power even when waveguided crystals are used [13 19]. Much more pump power is required for QFCs with bulk crystals or $\chi^{(3)}$ media due to shorter interaction time or smaller nonlinearity. This prevents the scalable integration of the photonic Rabi osillation for simulating more complex quantum systems. A promising approach for saving the cw pump power is an enhancement of optical nonlinearity by using an optical cavity. However, cavity systems in which all relevant lights are confined such as ring resonator systems 20, 21] severely limit accessible frequencies and bandwidths of the photons, which sacrifices the feature of the photonic Rabi oscillation.

In this study, without losing the acceptable frequencies and bandwidth, we demonstrate cavity enhancement of the photonic Rabi oscillation. We utilize a periodically-poled lithium niobate (PPLN) waveguide as a $\chi^{(2)}$ medium with a cavity only for the pump light (Fig. 1 (b)), which we call the PPLN waveguide resonator (PPLN/WR) hereafter. The internal enhancement factor is estimated to be over 10 compared with convential QFCs with achieving a maximum transition probability over $90 \%$. Such an efficient photonic Rabi oscillation realizes full cycle of the coherent rotation between the two frequency modes for the first time. This also enables an all-optical control of polarizing photons beyond the manipulation of frequency degree of freedom (DOF). Thanks to the broad bandwidth property over $100 \mathrm{GHz}$ of the PPLN waveguide [13], we can simultaneously perform the operation over several dozen frequency and polarizing modes forming hyper-entangled states on dense quantum frequency combs [22, 23].

We explain the quantum theory of $\chi^{(2)}$-based photonic Rabi oscillation between angular frequencies $\omega_{\mathrm{g}}$ and $\omega_{\mathrm{e}}$ driven by a pump light at an angular frequency $\omega_{\mathrm{p}}\left(=\omega_{\mathrm{e}}-\omega_{\mathrm{g}}\right)$ for the nonlinear optical interaction [13]. The subscripts of the two energy levels 'e' and ' $\mathrm{g}$ ' mean 'excited' and 'ground' frequencies. The energy level relevant to the process is shown in Fig. 1 (b). When the pump light is sufficiently strong, and the perfect phase matching condition is satisfied, the effective Hamiltonian is described by $H=i \hbar\left(g^{*} a_{\mathrm{e}}^{\dagger} a_{\mathrm{g}}-g a_{\mathrm{e}} a_{\mathrm{g}}^{\dagger}\right)$, where $a_{\mathrm{e}(\mathrm{g})}$ is an annihilation operator of the higher (lower) frequency mode. The effective coupling contant $g=|g| e^{i \phi}$ is proportional to the complex amplitude of the pump light with phase $\phi$. From this Hamiltonian, annihilation operators $a_{\mathrm{e}, \text { out }}$ and $a_{\mathrm{g}, \text { out }}$ for the higher and lower frequency modes from the nonlinear optical medium are described 

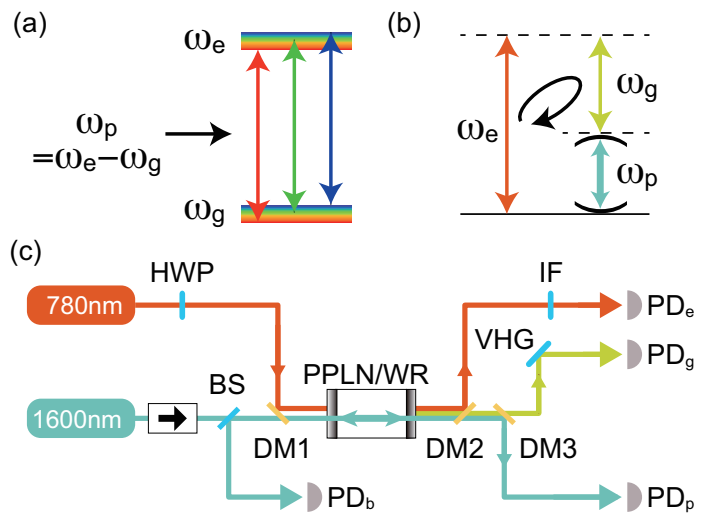

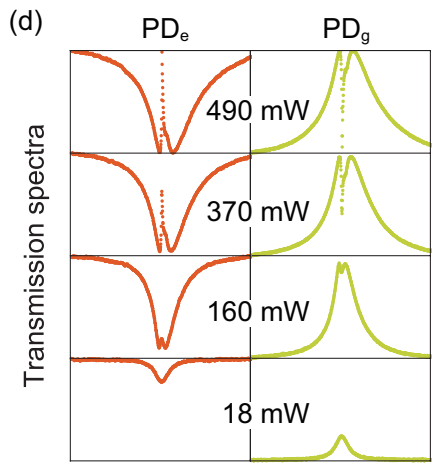

Pump frequency (a.u.) (e)

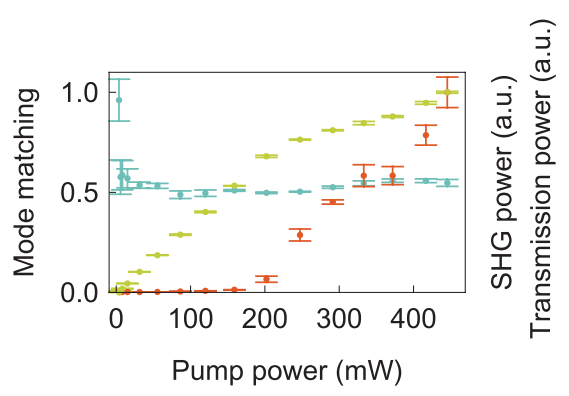

FIG. 1. (a) Two-level photonic systems coherently-coupled by photonic Rabi oscillation. Due to the virtual level structure, the ground and excited levels are regarded as continuously distributed. (b) Energy diagram of $\chi^{(2)}$ interaction equivalent to the coherent two-level systems. The pump light at angular frequency $\omega_{\mathrm{p}}$ is confined in the cavity. (c) Experimental setup. (d) Pump power dependencies of the transmission spectra of $780 \mathrm{~nm}$ light and $1522 \mathrm{~nm}$ light. The power in the figure is the pump power measured in front of the PPLN/WR. (e) Input pump power dependencies of the mode matching (coupling efficiency) of the pump light to the PPLN/WR (purple, left-hand axis), and SHG/transmission power of the pump light (red/brown, right-hand axis). The error bars indicate one standard deviation. For estimating the mode matching, we used the data with the standard deviations smaller than $5 \%$ (The leftest four data are omitted).

by

$$
\left[\begin{array}{c}
a_{\mathrm{e}, \text { out }} \\
a_{\mathrm{g}, \text { out }}
\end{array}\right]=\left[\begin{array}{cc}
\cos \frac{\theta}{2} & -e^{i \phi} \sin \frac{\theta}{2} \\
e^{-i \phi} \sin \frac{\theta}{2} & \cos \frac{\theta}{2}
\end{array}\right]\left[\begin{array}{c}
a_{\mathrm{e}} \\
a_{\mathrm{g}}
\end{array}\right]
$$

where $\theta / 2=|g| \tau$, and $\tau$ is the interaction time of the light through the nonlinear optical medium. This process is equivalent to the atomic Rabi oscillation driven by the external optical field resonant with the two energy levels. The branching ratio of the transition matrix can be adjusted by the pump power. We notice that at $\theta=2 \pi$, the light at the initial frequency mode is obtained with a unit probability, while $\pi$ phase shift understood by the geometric phase is added on its complex amplitude. When the nonlinear optical medium is placed inside a cavity confining only the pump light, the coupling strength $|g|$ is enhanced without any other modifications, resulting in a higher Rabi frequency while preserving the intrinsic bandwidth of the nonlinear interaction.

The experimental setup for the photonic Rabi oscillation between $780 \mathrm{~nm}$ and $1522 \mathrm{~nm}$ light driven by the cavity-enhanced pump light at $1600 \mathrm{~nm}$ is shown in Fig. 1(c). The cw pump light and the $780 \mathrm{~nm}$ light with a power of $1 \mathrm{~mW}$ are combined at a dichroic mirror (DM1), and then focused on the PPLN/WR.

The PPLN waveguide used in our experiment has a periodically-poling period for satisfying the type- 0 quasiphase-matching condition, and the polarization of the light relevant to the Rabi oscillation is $\mathrm{V}$. The length of the waveguide is $20 \mathrm{~mm}$. For forming the singly-resonant PPLN waveguide resonator with the Fabry-Pérot structure, the end faces of the waveguide are flat polished, and coated by dielectric multilayers. The reflectance for $1600 \mathrm{~nm}$ is about $98 \%$, and the quality factor of the cav- ity for the pump light is about $3.2 \times 10^{6}[23]$. For $780 \mathrm{~nm}$ and $1522 \mathrm{~nm}$, anti-reflective coatings are achieved with the reflectances of $5 \%$ and $0.1 \%$, respectively. The coupling efficiency of $780 \mathrm{~nm}$ light to the PPLN/WR is 0.91 .

After the PPLN/WR, the $780 \mathrm{~nm}$ light is reflected by DM2, and passes through an interference filter (IF) with a bandwidth of $3 \mathrm{~nm}$ followed by a photo detector $\left(\mathrm{PD}_{\mathrm{e}}\right)$. The $1522 \mathrm{~nm}$ light and the pump light pass through DM2, and they are separated by DM3. The $1522 \mathrm{~nm}$ light passing through DM3 is diffracted at a volume holographic grating (VHG) with a bandwidth of $1 \mathrm{~nm}$ and is detected by $\mathrm{PD}_{\mathrm{g}}$. The pump light reflected at DM3 is detected by $\mathrm{PD}_{\mathrm{p}}$. The pump light coming back from the PPLN/WR is monitored by $\mathrm{PD}_{\mathrm{b}}$.

The branching ratio between the two frequency modes characterized by rotation angle $\theta$ of Rabi oscillation in Eq. (1) was measured by the PDs for various pump powers while scanning the pump frequency. Examples of the observed power spectra for $780 \mathrm{~nm}$ and $1522 \mathrm{~nm}$ light are shown in Fig. 1 (d). We see that when the pump light was resonant to the PPLN/WR, the transition process was observed as a dip and a peak of the power spectra. For the pump power satisfying $0 \leq \theta \leq \pi$, a higher pump power leads to the deeper dip and the higher peak of the spectra (the bottom figure). For the pump power such that $\theta>\pi$ is satisfied (the other three figures), the dip at $780 \mathrm{~nm}$ and the peak at $1522 \mathrm{~nm}$ were respectively turned into upward and downward. This behavior shows the frequency recovery from $1522 \mathrm{~nm}$ to $780 \mathrm{~nm}$ after the transition from $780 \mathrm{~nm}$ to $1522 \mathrm{~nm}$.

The pump power was measured just before the PPLN/WR. In order to estimate an effective pump power $P$ used for the Rabi oscillation, we measured an optical 

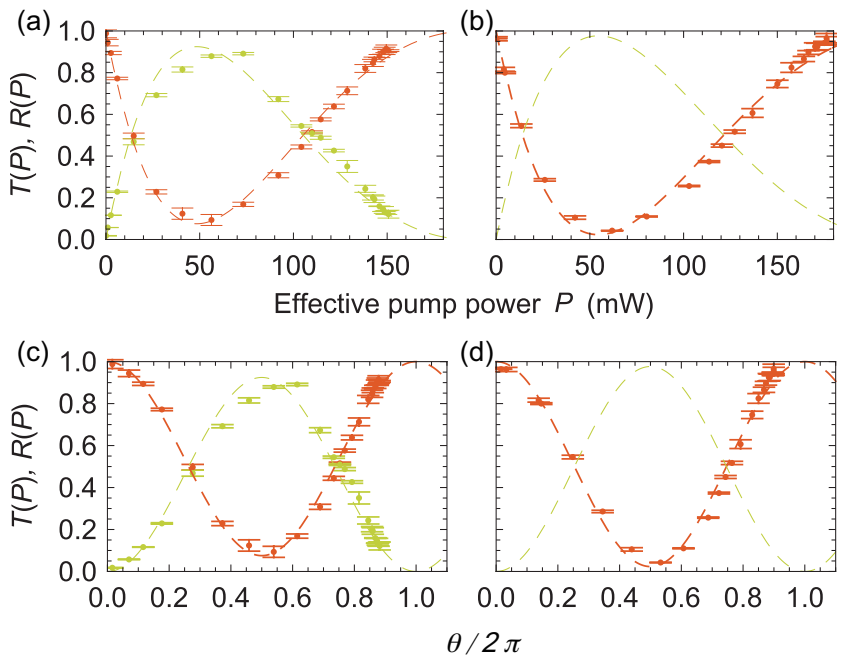

FIG. 2. (a) and (b) are for the signal light coming from the direction same as and opposite to the pump light. (c) and (d) show dependency on $\theta$ in the Rabi cycle instead of the effective pump power in (a) and (b).

mode matching (a coupling efficiency) to the resonator of the pump light from the reflection spectra at $\mathrm{PD}_{\mathrm{b}}$, and second harmonic generation (SHG) at $800 \mathrm{~nm}$ of the pump light at $\mathrm{PD}_{\mathrm{e}}$ as an unexpected nonlinear optical interaction. We show the experimental results in Fig. 1(e). From the figure, we estimated the amount of the mode matching to be 0.52 on average. The second harmonic light at $800 \mathrm{~nm}$ was measured by $\mathrm{PD}_{\mathrm{e}}$ without the $780 \mathrm{~nm}$ signal light and IF. In Fig. 1(e), we see that SHG power is gradually increased. Corresponding to this, the transmitted pump power measured by $\mathrm{PD}_{\mathrm{p}}$ begins to deviate from the proportional relationship with the input pump power. This indicates that the coupled pump power is consumed by SHG, and the transmitted power reflects the remaining pump power $P$ used for the frequency transition. From the experimental data for $<200 \mathrm{~mW}$ input pump power, in which SHG is negligibly small, we estimated the conversion factor between the transmitted pump power and $P$ with considering the mode matching 0.52 , and then we determined $P$ for all input pump power.

Together with the estimated values of $P$ and the observed spectra of $780 \mathrm{~nm}$ and $1522 \mathrm{~nm}$ light, we plot the staying probability (transmittance) $T(P)$ and the transition probability (reflectance) $R(P)(:=1-T(P))$ in Figs. 2 (a) and (c) based on the normalization method in Refs. [7, 24]. The maximum transition probability corresponding to the $\pi$ pulse was achieved at $\sim 50 \mathrm{~mW}$ pump power. The best fit to $T(P)$ with a function $1-$ $A \sin ^{2}(\sqrt{B P} L)$ gives $A=0.92$ and $B=0.13 \mathrm{~W}^{-1} \mathrm{~mm}^{-2}$, where $L=20 \mathrm{~mm}$ is the waveguide length. The observed coupling constant $B$ is over 10 times larger than previously-reported values in QFC experiments without
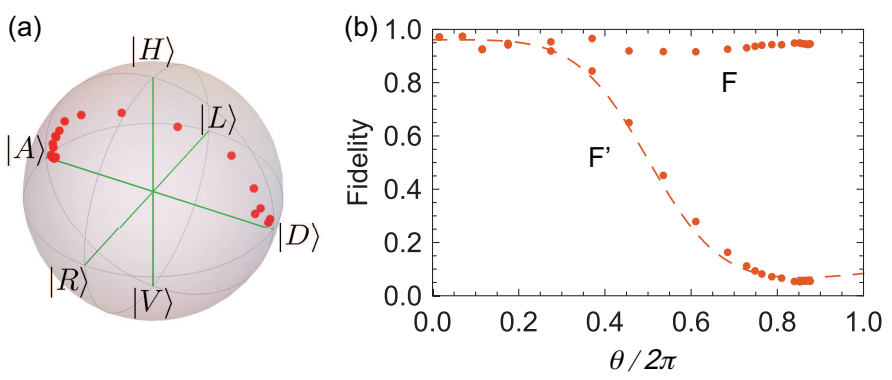

FIG. 3. (a) The Poincaré sphere (Bloch sphere) for the polarization state of light. $|R\rangle(|L\rangle)$ is the right (left) circular polarization state. The trajectory of the states from $\theta=0$ to $\theta \sim \pi$ is represented by red circles from near $|D\rangle$ to $|A\rangle$. (b) $\theta(P)$ dependencies of fidelities $F$ and $F^{\prime}$. The dotted curve is theoretically obtained by using experimental parameters.

cavity systems $13-19]$. As a result, the cavity enhancement effect was clearly observed. We will give the detailed discussion about the enhancement later.

The pump light confinement in the Fabry-Pérot cavity allows for the Rabi oscillation regardless of the input direction of the pump light. To see this, we swapped the positions of the $780 \mathrm{~nm}$ light source and the detector $\mathrm{PD}_{\mathrm{e}}$. The experimental result is shown in Fig. 2 (b) and (d). As is expected, the oscillation of the frequency transition was surely observed. The best fit to $T(P)$ with $1-A^{\prime} \sin ^{2}\left(\sqrt{B^{\prime} P} L\right)$ gives $A^{\prime}=0.98$ and $B^{\prime}=0.11 \mathrm{~W}^{-1} \mathrm{~mm}^{-2}$. These estimated values are similar to $A$ and $B$, and the bidirectional photonic Rabi oscillation was successfully achieved.

To see the amount of the cavity enhancement, we compare the value of the normalized coupling constant $B=0.13 \mathrm{~W}^{-1} \mathrm{~mm}^{-2}$ with the values in previous reported papers. In Ref. [13], the QFC system including the PPLN waveguide and wavelengths of the relevant light is almost the same as this experimental setup except for the cavity coating. The value of the coupling constant is calculated to be $0.009 \mathrm{~W}^{-1} \mathrm{~mm}^{-2}$ from the reported experimental data. From these results, an enhancement factor of the coupling constant is estimated to be 13 . While the value is slightly smaller than a theoretically predicted value 25, 26] $F / \pi \sim 19$ with finesse $F=59$ [23], our result surely showed that the coupling strength becomes an order of magnitude larger. This statement holds in comparison to other QFC experiments using PPLN waveguides without cavities in various situations [14-19] in which the coupling constant from 0.003 to $0.012 \mathrm{~W}^{-1} \mathrm{~mm}^{-2}$ were observed.

The photonic Rabi oscillation can be used for the polarization rotation of the signal light. The experiment for such the operation was performed by the same setup in Fig. 1 (c). The input signal light at $780 \mathrm{~nm}$ is set to diagonal polarization whose state is written as $|D\rangle:=(|H\rangle+|V\rangle) / \sqrt{2}$, where $|H\rangle$ and $|V\rangle$ are states 
for the horizontal and vertical polarization of light, respectively. Because only the $\mathrm{V}$-polarized light is frequency converted by the type-0 quasi phase-matched PPLN/WR, the polarization state of the light at $780 \mathrm{~nm}$ is transformed into $\left|\psi_{\theta}\right\rangle:=\mathcal{N}\left(|H\rangle+\cos \frac{\theta}{2}|V\rangle\right)$ ideally, where $\mathcal{N}:=\left(1+\cos ^{2} \frac{\theta}{2}\right)^{-1 / 2}$ is the normalization constant. For $780 \mathrm{~nm}$ light coming from the PPLN/WR, the polarization state tomography was performed by inserting a polarization analyzer composed of a quarter wave plate, a HWP and a polarizing BS into the optical path just before $\mathrm{PD}_{\mathrm{e}}$.

In Fig. 3 (a), the trajectory of the polarization states on Poincaré sphere (or Bloch sphere) estimated from Stokes parameters is shown. As the pump power increases, the initial state near $\left|\psi_{0}\right\rangle(=|D\rangle)$ is transformed towards $\left|\psi_{2 \pi}\right\rangle=|A\rangle:=(|H\rangle-|V\rangle) / \sqrt{2}$, via a state near $\left|\psi_{\pi}\right\rangle=|H\rangle$. For quantitative evaluation of the rotation, we borrowed the density operator representation $\rho_{\theta}$ of the polarization state for various values of $\theta=\theta(P)$ from the quantum information theory. We evaluated the fidelities $F:=\left\langle\psi_{\theta}\left|\rho_{\theta}\right| \psi_{\theta}\right\rangle$ of $\rho_{\theta}$ to the ideal state $\left|\psi_{\theta}\right\rangle$ and $F^{\prime}:=\left\langle\psi_{0}\left|\rho_{\theta}\right| \psi_{0}\right\rangle$ of the states with turning on and off the pump light. The results are shown in Fig. 3(b). For every $\theta, F$ is higher than 0.9 , and thus the polarization state is successfully rotated by the Rabi oscillation for any pump power with a resonant frequency. The result of $F^{\prime}$, which approaches zero as $\theta$ increases, also shows the rotation effect. We notice that for $\theta=2 \pi, F^{\prime}$ is close to zero due to the effect of the geometric phase added through the full Rabi cycle [27-29]. The above polarization rotation was controlled by amplitude modulation (AM) of the pump light at a frequency resonant on the cavity. In addition to the AM control, the cavity structure enables us to toggle the polarization rotation by frequency modulation (FM) of the pump light between off-resonant and on-resonant condition with a fixed pump power.

We construct a theoretical model for the state transformation by the photonic Rabi oscillation. The observed maximum transition efficiency is $A=0.95$. We assume that the imperfection is originated from the propagation mode mismatch of the signal and the pump light. We denote the propagation mode which interacts with the pump light by $|x\rangle$ and its orthogonal mode by $|\bar{x}\rangle$. Under the assumption, when the input state is a pure state with diagonal polarization, the initial state to QFC can be written as $\left|\psi_{\text {in }}\right\rangle:=|D\rangle(\sqrt{A}|x\rangle+\sqrt{1-A}|\bar{x}\rangle)$. After the Rabi oscillation with $\theta$, the normalized output state $\left|\psi_{\text {out }}\right\rangle$ is ideally described by

$$
\left|\psi_{\text {out }}\right\rangle:=\mathcal{N}_{0}\left(\sqrt{A} \mathcal{N}^{-1}\left|\psi_{\theta}\right\rangle|x\rangle+\sqrt{2(1-A)}|D\rangle|\bar{x}\rangle\right),
$$

where $\mathcal{N}_{0}:=\left(A \mathcal{N}^{-2}+2(1-A)\right)^{-1 / 2}$ is a normalization factor. In our experiment, the reconstructed states $\rho_{\theta}$ were slightly impure, namely $\operatorname{tr}\left(\rho_{\theta}^{2}\right)<1$. We model the output state including the impurity as $\rho_{\mathrm{th}}:=$ $p\left|\psi_{\text {out }}\right\rangle\left\langle\psi_{\text {out }}\right|+(1-p) I / 2$, where $p:=\left(2\left\langle\operatorname{tr}\left(\rho_{\theta}^{2}\right)\right\rangle-1\right)^{1 / 2}$ and $I$ is the identity operator. By using experimentally observed values $A=0.95$ and $\left\langle\operatorname{tr}\left(\rho_{\theta}^{2}\right)\right\rangle=0.93$, we obtained the fidelity $F_{\text {th }}:=\left\langle\psi_{\text {in }}\left|\rho_{\text {th }}\right| \psi_{\text {in }}\right\rangle$ as shown in Fig. 3 (b). We see that the experimental results are in good agreement with the curve theoretically predicted with the use of the experimental parameters.

In our demonstration for the polarization state rotation, we measured only the polarization state of $780 \mathrm{~nm}$ light. When we consider the frequency DOF composed of $780 \mathrm{~nm}$ and $1522 \mathrm{~nm}$ modes, which we denote by $\left|\omega_{\mathrm{h}}\right\rangle$ and $\left|\omega_{1}\right\rangle$, the photonic Rabi oscillation system works on a single-photon two-qubit state 30] formed by the polarization and the frequency modes of the single photon. Because the PPLN/WR has polarization dependency, an input state $|D\rangle\left|\omega_{\mathrm{h}}\right\rangle$ is deterministically transformed into the single-photon Bell states as $\left(|H\rangle\left|\omega_{\mathrm{h}}\right\rangle \pm|V\rangle\left|\omega_{\mathrm{l}}\right\rangle\right) / \sqrt{2}$ by the Rabi oscillation with $\theta=\pi / 4$ and $3 \pi / 4$. These operations correspond to the controlled NOT gates. Combining frequency dependent WPs, the other two Bell states can be easily generated. The measurement of the four Bell states is also deterministically achieved by the same setup.

In conclusion, we have demonstrated cavity enhancement of photonic Rabi oscillation while keeping the flexible choice of frequencies and wide acceptance bandwidths of photons. The enhancement of the nonlinear optical coupling was 10 times larger than convential frequency converters. This leads to the observation of the full Rabi cycle between the two photonic frequencies, resulting in all-optical versatile manipulation beyond on the frequency DOF. Our results will open up a large-scale photonic quantum information processing based on frequency modes including hyper-entangled systems [31, 32]. Furthermore, considering more than two virtual energy levels and multiple pump lasers, the photonic system will enable to simulate and design the complicated atomic systems.

RI, NI and TY acknowledge members of Quantum Internet Task Force (QITF) for comprehensive and interdisciplinary discussions of the Quantum Internet. This work was supported by CREST, JST JPMJCR1671; MEXT/JSPS KAKENHI Grant Number JP20H01839 and JP18K13483; Asahi Glass Foundation.

[1] W. McGrew et al., Nature 564, 87 (2018).

[2] K. Bongs et al., Nature Reviews Physics , 1 (2019).

[3] H. J. Kimble, Nature 453, 1023 (2008).

[4] J. I. Cirac and P. Zoller, Nature 404, 579 (2000).

[5] I. S. Madjarov et al., Nature Physics , 1 (2020).

[6] P. Kumar, Optics Letters 15, 1476 (1990).

[7] T. Kobayashi et al., Nature Photonics 10, 441 (2016).

[8] S. Clemmen, A. Farsi, S. Ramelow, and A. L. Gaeta, Phys. Rev. Lett. 117, 223601 (2016).

[9] C. Joshi et al., Phys. Rev. Lett. 124, 143601 (2020). 
[10] M. Y. Niu, I. L. Chuang, and J. H. Shapiro, Phys. Rev. Lett. 120, 160502 (2018).

[11] M. Y. Niu, I. L. Chuang, and J. H. Shapiro, Phys. Rev. A 97, 032323 (2018)

[12] S. Krastanov et al., arXiv preprint arXiv:2002.07193 (2020).

[13] R. Ikuta et al., Nature Communications 2, 537 (2011).

[14] B. Albrecht, P. Farrera, X. Fernandez-Gonzalvo, M. Cristiani, and H. De Riedmatten, Nature communications 5, 3376 (2014).

[15] R. Ikuta et al., Nature Communications 9, 1997 (2018).

[16] M. Bock et al., Nature communications 9, 1998 (2018).

[17] A. Dréau, A. Tchebotareva, A. E. Mahdaoui, C. Bonato, and R. Hanson, Phys. Rev. Applied 9, 064031 (2018).

[18] Y. Yu et al., Nature 578, 240 (2020).

[19] T. van Leent et al., Phys. Rev. Lett. 124, 010510 (2020).
[20] X. Guo, C.-L. Zou, H. Jung, and H. X. Tang, Phys. Rev. Lett. 117, 123902 (2016).

[21] X. Lu et al., Nature Photonics 13, 593 (2019).

[22] M. Kues et al., Nature Photonics 13, 170 (2019).

[23] R. Ikuta et al., Phys. Rev. Lett. 123, 193603 (2019).

[24] R. Ikuta et al., Optics Express 21, 27865 (2013).

[25] R. Regener and W. Sohler, JOSA B 5, 267 (1988).

[26] M. Stefszky et al., Journal of Optics 20, 065501 (2018).

[27] A. P. VanDevender and P. G. Kwiat, Optics Express 15, 4677 (2007).

[28] N. K. Langford et al., Nature 478, 360 (2011).

[29] A. Karnieli, S. Trajtenberg-Mills, G. Di Domenico, and A. Arie, Optica 6, 1401 (2019).

[30] Y.-H. Kim, Phys. Rev. A 67, 040301 (2003).

[31] J. T. Barreiro, T.-C. Wei, and P. G. Kwiat, Nature physics 4, 282 (2008).

[32] C. Reimer et al., Nature Physics 15, 148 (2019). 